\title{
Iteration in ACL2
}

\author{
Matt Kaufmann and J Strother Moore \\ Department of Computer Science \\ The University of Texas at Austin, Austin, TX, USA \\ $\{$ kaufmann, moore\}@cs . utexas.edu
}

\begin{abstract}
Iterative algorithms are traditionally expressed in ACL2 using recursion. On the other hand, Common Lisp provides a construct, loop, which - like most programming languages - provides direct support for iteration. We describe an ACL2 analogue loop\$ of loop that supports efficient ACL2 programming and reasoning with iteration.
\end{abstract}

\section{Introduction}

Recursion is a natural way to define notions in an equational logic. But for programming, iteration is often more natural and concise than recursion.

This paper introduces an ACL2 iteration construct, loop\$. We give an overview of its usage in programming and reasoning, and we touch on some key aspects of its implementation. Those who want more complete user-level documentation are welcome to see :DOC loop\$1, and those who want more implementation details may visit Lisp comments in the ACL2 sources, in particular the "Essay on Loop\$" and the "Essay on Evaluation of Apply\$ and Loop\$ Calls During Proofs".

The rest of this introduction illustrates the basics of loop $\$$ and then mentions some related prior work. Next, Section 2 outlines the syntax of loop\$ expressions. Section 3 provides necessary background on apply $\$$ and especially warrants. Then Section 4 gives semantics to loop $\$$ expressions by translating them into the logic, which provides background for the discussion of reasoning about loop\$ in Section 5. Section [ discusses evaluation of calls of loop\$, including considerations involving guards. We conclude by considering possible future enhancements to loop $\$$.

The community book projects/apply/loop-tests.lisp constitutes supporting materials for this paper: it contains most ACL2 events and expressions discussed below.

\subsection{Loop $\$$ basics}

Common Lisp supports iteration with the construct loop, and ACL2 provides the analogous (though less general) construct loop $\$ 2$ These two forms evaluate to 30, in Common Lisp and in ACL2, respectively.

(loop for $\mathrm{x}$ in '( 12234$)$ sum $(* x \mathrm{x})$ )

(loop $\$$ for $x$ in '( $\left(\begin{array}{llll}1 & 2 & 3 & 4\end{array}\right)$ sum $(* x x)$ )

A call of loop $\$$ is given semantics by translating it to a logical translated term that is a call of a loop $\$$ scion, in the spirit of a higher-order function call. For example the translation of the loop $\$$ form above is the following call of the loop\$ scion, sum\$, whose first argument is a quoted lambda object.

\footnotetext{
${ }^{1}$ In the online version of this paper, we follow the usual convention of adding links to documentation on the web [4] Sometimes we say "see :DOC topic", but other times we simply underline a documentation topic.

${ }^{2}$ The dollar sign is commonly used as a suffix in ACL2 to distinguish from a similar Common Lisp utility. Note that the symbol loop in the "COMMON-LISP" package is one of the 978 external symbols in that package that are imported into the "COMMON-LISP" package, so we cannot distinguish acl2: : loop from common-lisp: :loop; these are the same symbol.
}

G. Passmore, R. Gamboa (Eds.): ACL2 Workshop 2020 EPTCS 327, 2020, pp. 16 31 doi 10.4204/EPTCS.327.2 (c) M. Kaufmann \& J S. Moore

This work is licensed under the Creative Commons Attribution License. 


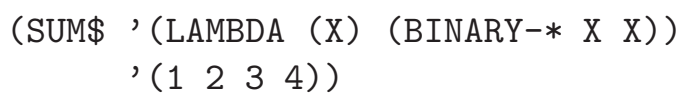

Sum\$ has the following definition, ignoring declarations and calls of mbe and fix.

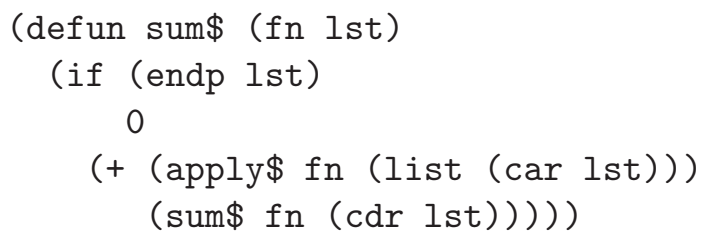

Notice the use of apply\$, to apply a given function symbol or lambda object to a list of arguments. Support for loop $\$$ thus depends on the sort of "higher-order" capability provided by apply\$. (We return to apply $\$$ in Section 3 .)

In the following example we use a different loop $\$$ scion, collect $\$$, to collect into a list instead of summing results. Also, we filter by considering only even numbers that are multiples of 5 (thus: multiples of 10), stopping when we hit a number greater than 30 .

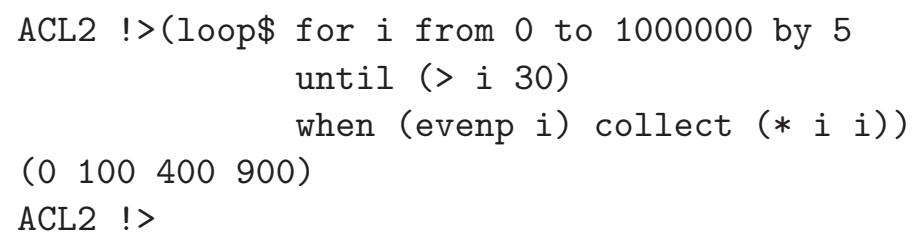

This time we get essentially the following translation of the loop $\$$ form. Reading it bottom-up (insideout), we see that from-to-by builds a list of integers $\left(\begin{array}{llllll}0 & 5 & 10 & 15 & \ldots & 1000000\end{array}\right)$, which until $\$$ reduces to ( $\left.\begin{array}{lllllll}0 & 5 & 10 & 15 & 20 & 25 & 30\end{array}\right)$ by stopping when 30 is exceeded; then when $\$$ restricts that list to the even integers to produce the list ( $\left.\begin{array}{llll}0 & 10 & 20 & 30\end{array}\right)$, and finally collect $\$$ assembles the squares of the members of that list to produce the result, ( $\begin{aligned} & 0 \\ & 100\end{aligned} 400$ 900). We believe that this compositional, though computationally inefficient, formal semantics is useful for reasoning about applications of loop\$ since lemmas can be proved to simplify the respective steps.

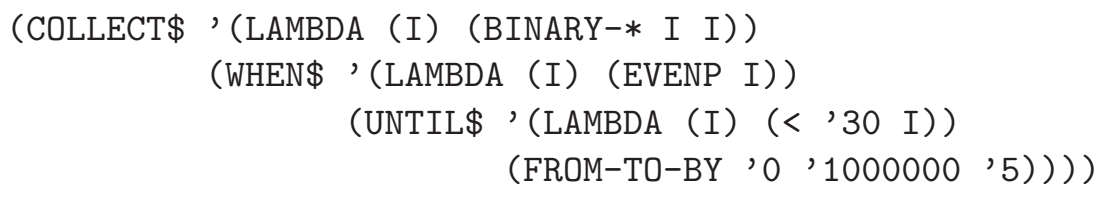

Loop\$ scions (including collect\$, when\$, and until\$) repeatedly call apply\$ on a given lambda object (or function symbol). In Section 3 we explore consequences of these uses of apply $\$$.

If we define a guard-verified function using a corresponding call of loop $\$$, then evaluation is more efficient. Consider the following variation of the example just above, which adds an of-type clause to aid in guard verification (more on that is in Section 6). The include-book event shown is important for almost all work that involves apply $\$$ or loop $\$$.

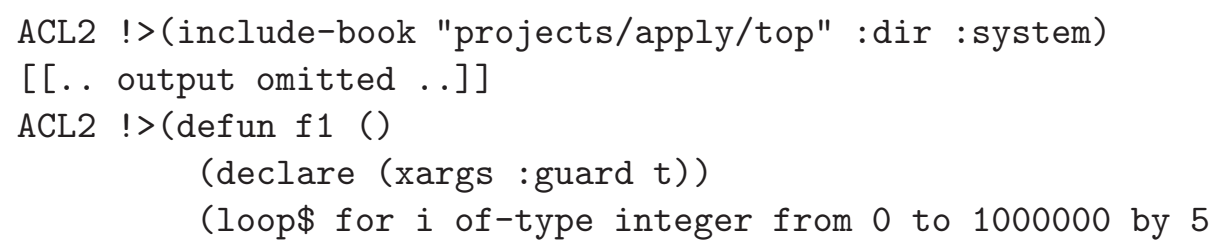




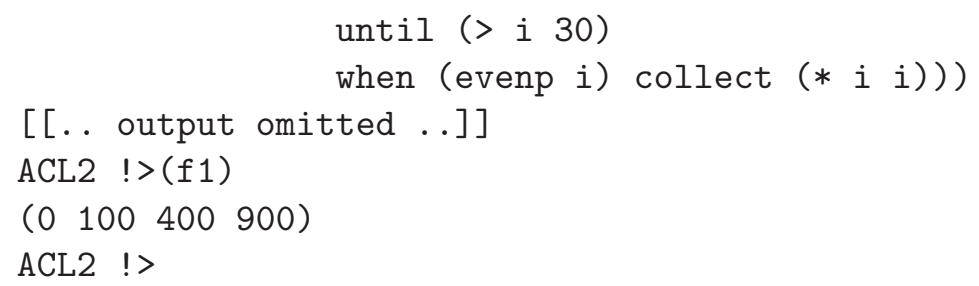

After $\mathrm{f} 1$ is admitted with guards verified, its Common Lisp definition is used for evaluation (as usual). The Common Lisp macroexpansion of the loop $\$$ form above is essentially as follows, which presumably allows the Common Lisp compiler to generate efficient code, in particular avoiding the indirection of apply $\$$, the repeated passes implied by our compositional semantics, and (presumably) use of the heap to form the intermediate lists noted above.

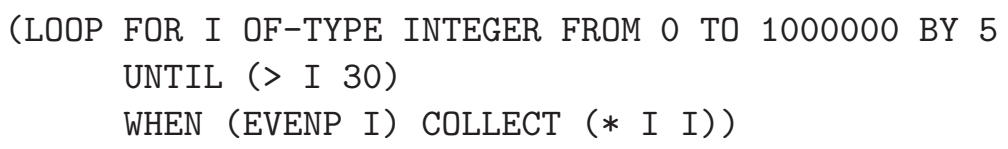

\subsection{Before ACL2}

The addition of an iteration construct within the first-order logic of the Boyer-Moore provers has been a longstanding goal. It was first explored by Moore in 1975 [6]. The Nqthm prover [2] of Boyer and Moore provided an iterative construct, called FOR, which provided simple loops such as

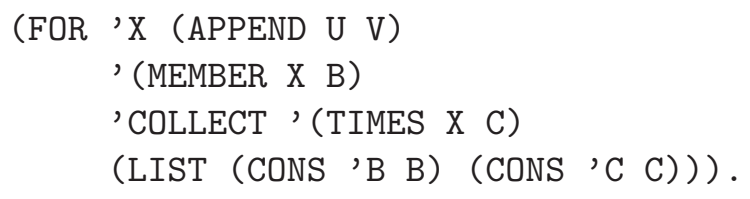

The expression above can be written in Common Lisp as

(loop for $\mathrm{x}$ in (append $\mathrm{u} \mathrm{v}$ ) when (member $\mathrm{x} b$ ) collect $(* \mathrm{x} \mathrm{c})$ )) .

Nqthm's FOR was defined using a universal interpreter for Nqthm called V\&C $\$$ and many familiar theorems about FOR were proved by Nqthm [1]. However, V\&C $\$$ was a non-constructive function [5] and, in addition, was not compatible with local events; Nqthm, unlike ACL2, did not support local. Thus V\&C \$ was abandoned for ACL2.

\section{Syntax}

The syntax of loop $\$$ is a restriction of the syntax of Common Lisp's loop macro:

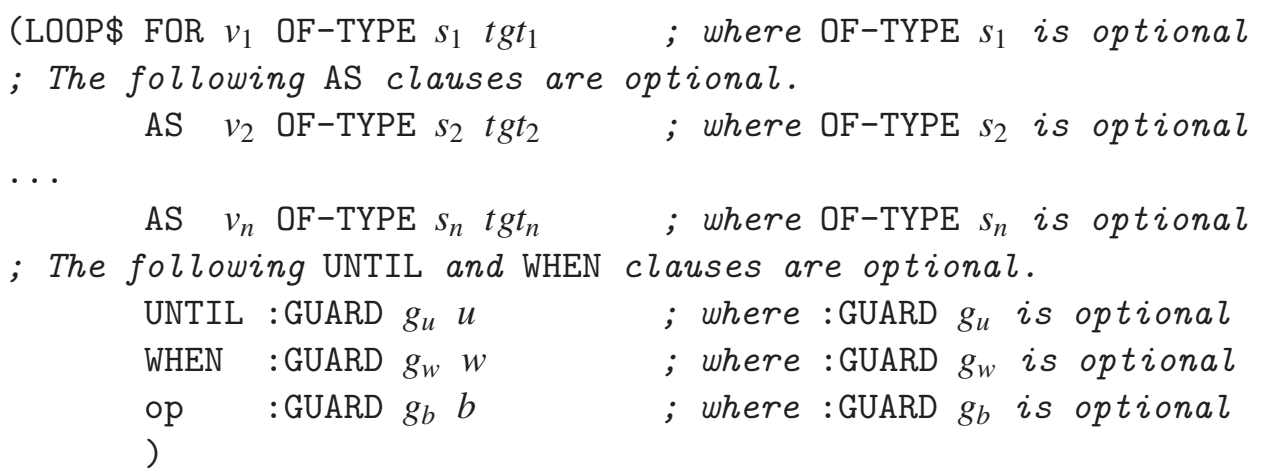


where the $v_{i}$ are distinct variables, the iteration variables; each OF-TYPE $s_{i}$ is optional where $s_{i}$ is a type-spec; each tgt $_{i}$ is a target clause (see below); each of $g_{u}, u, g_{w}, w, g_{b}$, and $b$ is a term; and op is one of the loop operators ALWAYS, THEREIS, APPEND, COLLECT, or SUM. We call $b$ the loop body. See :DOC loop\$ for restrictions, e.g., on terms being in :logic mode and tame — where user-defined function symbols all have warrants (see Section 3) - and on not allowing both a WHEN clause and an ALWAYS or THEREIS operator.

A target clause has one of the following forms, where $l s t$ is a term denoting a list, $l o$ and $h i$ are terms denoting integers, and the step expression $s$ (if supplied) is a term denoting a positive integer.

- IN $l s t$

- ON lst

- FROM lo TO hi

- FROM lo TO hi BY $s$

The corresponding target list is $l s t$ in the first case, the list of non-empty tails of $l s t$ in the second, the list of integers $(l o l o+1 \ldots h i)$ in the third case, and the list of integers $(l o l o+s \ldots)$ terminating just before exceeding $h i$ in the fourth case.

\section{Apply\$ and Warrants}

All of the loop\$ scions are defined using calls of apply $\$$. Thus, the value of a loop $\$$ expression depends on values of apply $\$$ calls. If $F$ is a function symbol of arity $n$, then it is clearly desirable for both rewriting and evaluation to replace a call (apply\$ ' $F$ (list $\left.t_{1} \ldots t_{n}\right)$ ) by $\left(F t_{1} \ldots t_{n}\right)$. But for user-defined $F$, the equality of these two terms depends on the warrant hypothesis for $F$ : the assertion (apply\$-warrant-F), where apply\$-warrant-F is the warrant of $F$. The interested reader may find further background on apply $\$$, including logical issues about the need for warrants, in our paper on apply \$ [3] and in discussions in :DOC apply\$, :DOC warrant, and (regarding the so-called "local problem") :DOC introduction-to-apply\$.

The example below illustrates warrants and warrant hypotheses. We begin as follows where as noted above, the include-book event is important for almost all work that involves apply $\$$ or loop\$.

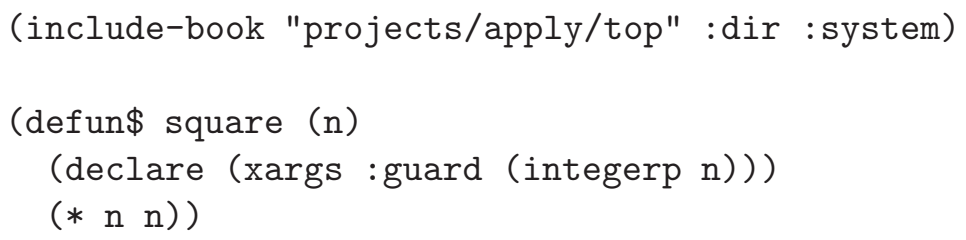

A call of defun\$ generates a corresponding defun event and introduces the warrant for square, which is the function symbol apply\$-warrant-square, with the event (defwarrant square). The following rewrite rule, named apply $\$$-square, is the key property of the warrant hypothesis, which is forced here so that a proof may progress to the forcing round as described below.

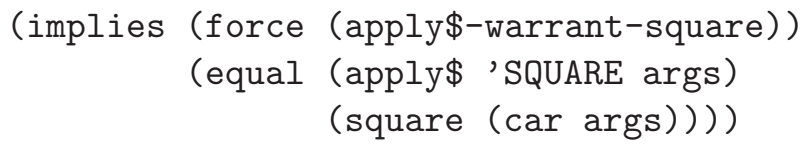


We may abbreviate (apply $\$$-warrant-square) with the macro call, (warrant square). More generally, for a function symbol $F$, (warrant $F$ ) abbreviates (apply $\$$-warrant-F). We see that the rewrite rule above replaces a call of apply $\$$ on 'SQUARE by the corresponding call of square.

In summary: the warrant hypothesis for $F$ justifies replacing (apply $\$$ ' $F$ ( I ist $\left.t_{1} \ldots t_{n}\right)$ ) by $(F$ $\left.t_{1} \ldots t_{n}\right)$.

The following sequence of events, extending the include-book and defun $\$$ forms displayed above, illustrates the role of warrant hypotheses.

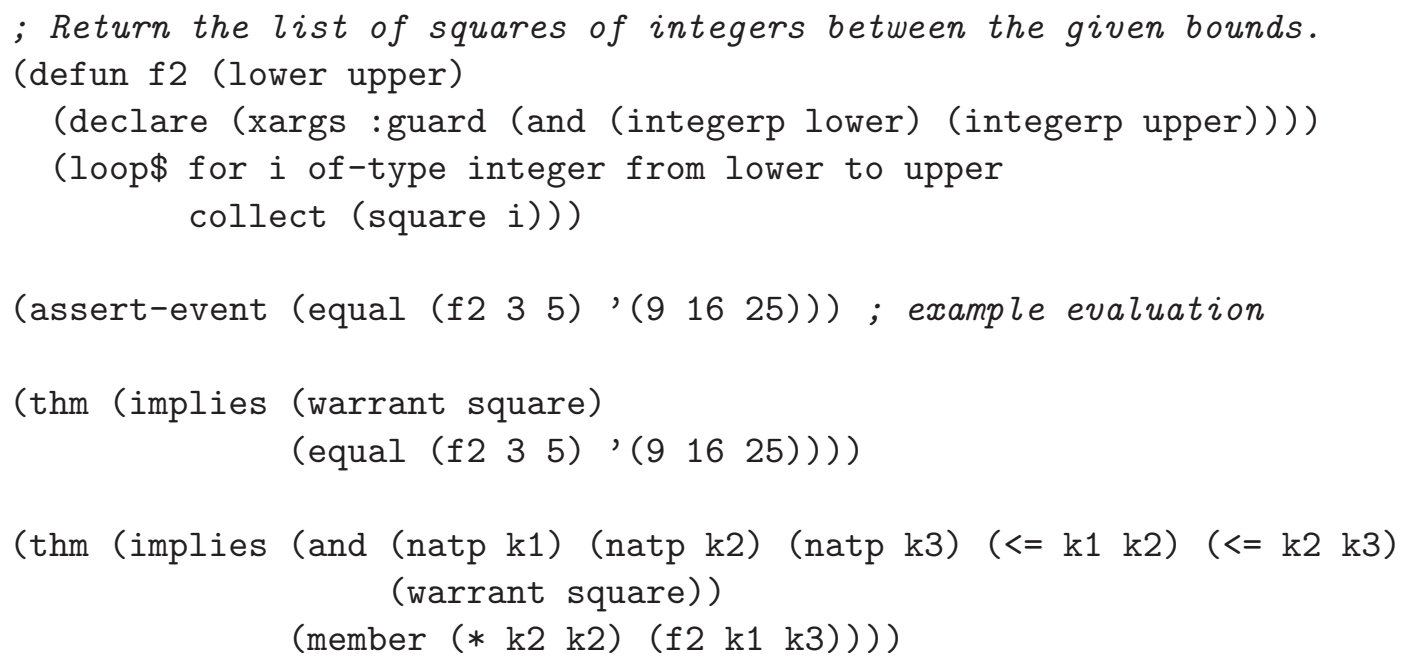

If the warrant hypothesis is omitted in the two calls of thm above, the proofs will progress to forcing rounds before failing. The forced goals make clear the need for a warrant hypothesis. For example, if the warrant hypothesis is removed before submitting the first thm form above, then the forcing round has the goal (APPLY\$-WARRANT-SQUARE) 3

Note that proofs succeed automatically for both thm forms above. They succeed without the initial include-book event and without any warrant hypotheses if we replace (square $\mathrm{x}$ ) by $(* \mathrm{x} \mathrm{x})$ in the definition of $f 2$; only user-defined functions have warrants (or require warrant hypotheses), not primitives like $*$ (more precisely, binary-*; * is a macro).

\section{Semantics: Translation to Logic}

The introduction presented the example

(loop\$ for $\mathrm{x}$ in '( $\left.\begin{array}{llll}1 & 2 & 3 & 4\end{array}\right)$ sum $(* \mathrm{x} x)$ )

and stated that it "essentially" translates to the following term.

$\begin{aligned}(\text { SUM } \$ & ,(\text { LAMBDA (X) (BINARY-* X X) ) } \\ & \left.,\left(\begin{array}{llll}1 & 2 & 3 & 4\end{array}\right)\right)\end{aligned}$

In this section we explain precisely how the simplest, plain loop $\$$ expressions such as this one are translated into loop $\$$ scion calls. We then discuss the translation of more complex fancy loop $\$$ expressions. We conclude with a note about relaxation of some restrictions for loop $\$$ expressions in theorems.

\footnotetext{
${ }^{3}$ Missing warrants from otherwise provable theorems do not necessarily lead to forcing because the proof may fail before then.
} 


\subsection{Plain loops and lambda $\$$}

A plain loop is a loop $\$$ expression that has a single iteration variable - that is, a FOR clause but no AS clause - which is the only variable that occurs free in the UNTIL test, WHEN test, or loop body. ACL2 gives semantics to a plain loop by generating a call of the loop $\$$ scion corresponding to the loop operator, where the first argument is a lambda\$ expression whose single formal is the iteration variable and whose body is the loop body. A lambda $\$$ expression, in turn, represents a corresponding quoted lambda object whose body is a translated term. Finally, additional calls of return-last are inserted (see below).

Let's see how this works on the loop $\$$ expression in the following definition.

(defun sum-squares (lst)

(loop\$ for $\mathrm{x}$ in lst $\operatorname{sum}(* \mathrm{x} \mathrm{x}))$ )

This is a simple loop: it binds only the variable $\mathrm{x}$, which is the only variable occurring free in the (translation of) the loop body. ACL2 first transforms this loop\$ expression to

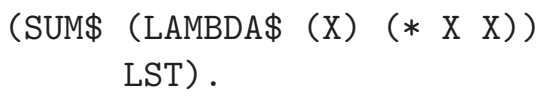

We see that the loop operator SUM generates a call of the corresponding loop $\$$ scion, sum $\$$. The first argument of that call is the lambda $\$$ expression whose single formal is the iteration variable, $\mathrm{x}$, and whose body is exactly the loop body. This lambda $\$$ expression, in turn, is transformed to a quoted lambda object by replacing the loop body $(* \mathrm{x} \mathrm{x})$ with its translation (binary-* $\mathrm{x} \mathrm{x}$ ), and by declaring that the formals - in this case, the single formal, $\mathrm{x}$ - may be ignored. (Such a declaration permits loop bodies that do not mention all of the iteration variables.)

(SUM\$ ' (LAMBDA (X)

(DECLARE (IGNORABLE $X)$ )

(BINARY-* X X))

LST)

But we're not done yet! The body of the lambda $\$$ expression is actually replaced by the translation of $($ prog2\$ ' (LAMBDA $\$(X)(* \quad X \quad X))(* x \quad x))$ - the system uses that quoted lambda\$ expression for efficient execution of compiled lambda objects 4 - and the loop $\$$ expression similarly is wrapped with a call of prog $2 \$$ that preserves the original loop $\$$ expression in the first argument, as discussed below in Subsection 6.2. Here is the translation of the body of sum-squares.

(RETURN-LAST 'PROGN

' (LOOP\$ FOR X IN LST SUM $(* X X))$

(SUM\$ ' (LAMBDA (X)

(DECLARE (IGNORABLE X))

(RETURN-LAST 'PROGN

' ( LAMBDA $\$(\mathrm{X})(* \mathrm{X} \mathrm{X}))$

$($ BINARY-* X X)) )

LST) )

Fortunately, one does not generally see such complexity during a proof. One reason is that the return-last calls are removed; see :DOC guard-holders. Another reason is that during a proof one

\footnotetext{
${ }^{4}$ See ACL2 source file apply-raw.lisp for details, in particular, the "Essay on the CL-Cache Implementation Details".
} 
sees untranslated terms (see :DOC term), and the untranslation process typically restores the original lambda $\$$ expression, so that in this case one sees ( $\operatorname{sum} \$(\operatorname{lambda} \$(\mathrm{x})(* \mathrm{x} x))$ lst).

We remark that a lambda $\$$ expression is only legal when, roughly speaking, it is ultimately used only as the first argument of apply $\$$ calls 5

\subsection{Fancy loops}

We explain translation of fancy loops using the following example. This definition contains a fancy loop both because the variables $\mathrm{m}$ and $\mathrm{n}$ are not bound by the loop $\$$ expression and because of the AS clause.

(defun $\mathrm{g}$ ( $\mathrm{m} \mathrm{n}$ lst1 lst2)

(loop $\$$ for $\mathrm{x} 1$ in 1 st1 as $\mathrm{x} 2$ in 1 st2 $\operatorname{sum}(* \mathrm{~m} \mathrm{n} \mathrm{x} 1 \mathrm{x} 2))$ )

For our discussion of fancy loops we will focus on basic semantics, ignoring return-last calls and ignorable declarations discussed for plain loops above.

Before we show the (essential) translation of the fancy loop above, we introduce auxiliary functions, loop\$-as and sum\$+. Loop\$-as is given a list L of $n$ lists and returns the sequence of $n$-tuples containing corresponding elements from each list until the shortest list is exhausted. For example:

ACL2 !>(loop\$-as ' (( $\left.\left.\begin{array}{lllllll}1 & 2 & 3 & 4 & \text { A } & \text { B C }\end{array}\right)\left(\begin{array}{llll}5 & 6 & 7 & 8\end{array}\right)\right)$ )

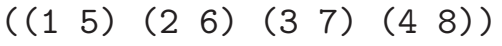

ACL2 !>

We next consider the fancy roop\$ scion sum $\$+$, which is called in the translation of the fancy loop above. Its first parameter, $\mathrm{fn}$, is expected to be a function symbol or lambda object with two arguments. The first argument of $\mathrm{fn}$ is expected to be a list of globals: values of the variables in the loop body that are not iteration variables. The second argument of $\mathrm{fn}$ is expected to be a list of locals: the result of a loop $\$$-as call, which (again) is a list whose $n^{\text {th }}$ member lists the $n^{\text {th }}$ iteration values of the iteration variables. Thus sum $\$+$ is defined much like sum $\$$ (here we ignore declarations as well as mbe and $\mathrm{fix}$ wrappers), but where the globals are passed when applying $\mathrm{fn}$ and the locals are wrapped into a list.

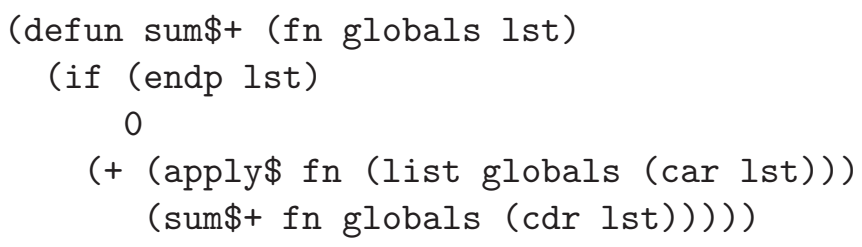

The fancy loop in the definition of $\mathrm{g}$, above, has the following semantics (translation). We see that the first argument is indeed a function of globals and locals, where the globals is the list containing the values of formals $\mathrm{m}$ and $\mathrm{n}$ of the function $\mathrm{g}$ defined above and the locals come from the loop $\$$-as call.

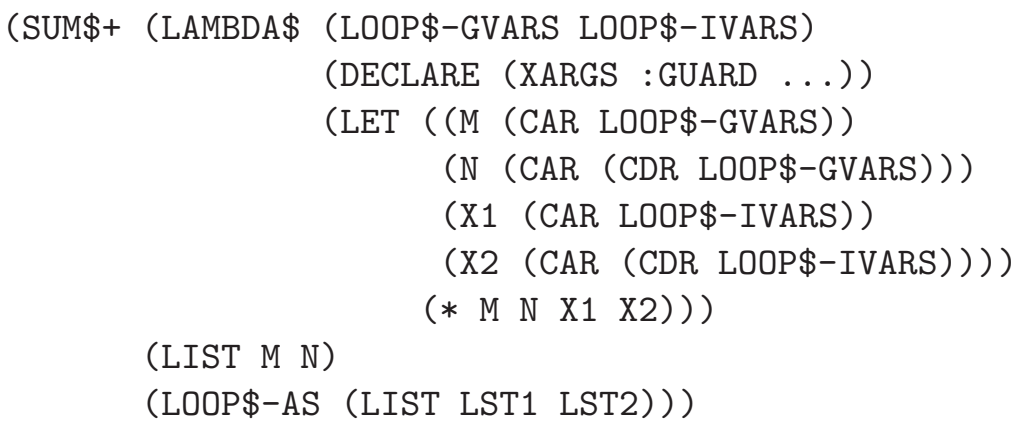

\footnotetext{
${ }^{5}$ To be precise, it must be in a position of ilk :FN [3].
} 
We have seen that the loop $\$$ operator, SUM, produces a call of a loop $\$$ scion: sum $\$$ for a plain loop and sum $\$+$ for a fancy loop. More generally, a loop $\$$ scion arises from any use of loop $\$$, and the scions introduced are all plain or all fancy depending on the particulars of the loop $\$$. The plain ones are sum $\$, \operatorname{collect} \$$, always $\$$, thereis $\$$, append $\$$, until $\$$, and when $\$$, respectively, and their fancy counterparts are sum $\$+$, collect $\$+$, always $\$+$, thereis $\$+$, append $\$+$, until $\$+$, and when $\$+$. The plain loop $\$$ scions may be summarized as follows, where the elements of the true-list lst are $e_{1}, \ldots, e_{n}$.

- ( $\operatorname{sum} \$$ fn lst): sums all (apply\$ fn (list $\left.e_{i}\right)$ )

- (always $\$$ fn lst): tests that all (apply $\$$ fn (list $\left.e_{i}\right)$ ) are non-nil

- (thereis $\$ \mathrm{fn}$ lst): returns the first non-nil value of (apply $\$$ fn $\left(\right.$ list $\left.\left.e_{i}\right)\right), i=1,2, \ldots, n$

- (collect $\$$ fn lst): conses together all (apply $\$$ fn (list $\left.e_{i}\right)$ )

- (append $\$$ fn lst): appends together all (apply $\$$ fn (list $\left.e_{i}\right)$ )

- (until\$ fn lst): lists all $e_{i}$, in order, until the first $i$ such that (apply $\$$ fn (list $\left.e_{i}\right)$ ) is non-nil

- (when $\$$ fn lst): lists those $e_{i}$, in order, such that (apply $\$$ fn (list $e_{i}$ )) is non-nil

The fancy loop\$ scions are analogous. We have already seen the definition of sum $\$$, and we have seen a similar definition of sum $\$+$ that accommodates the globals. Here is an analogous pair of definitions for when $\$$ and when $\$+$.

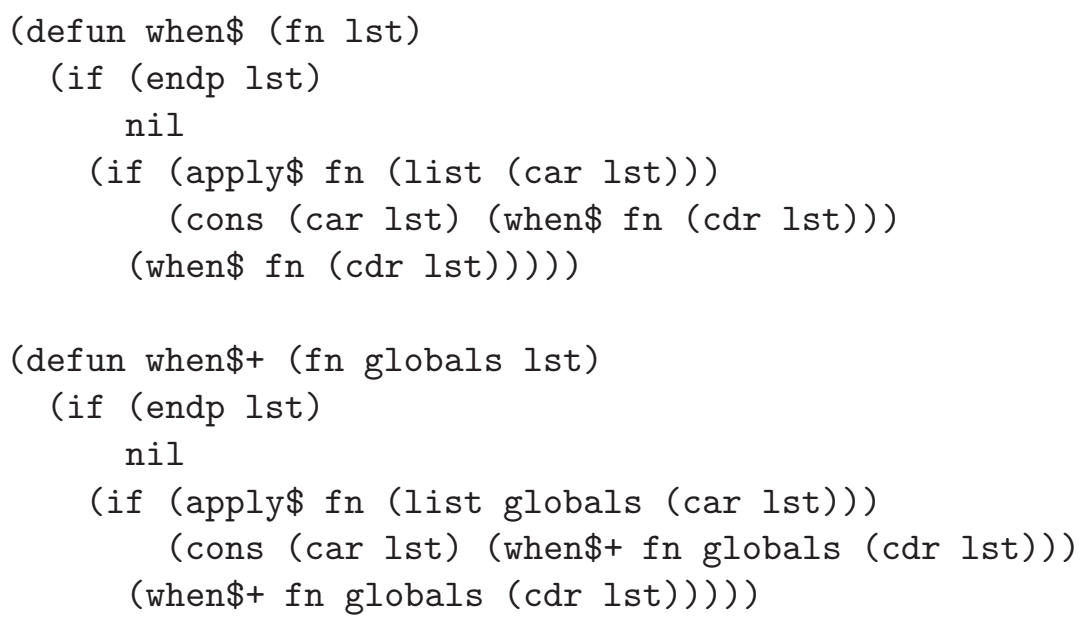

\subsection{Loop $\$$ restrictions are eased in theorems}

Conventional syntactic requirements are enforced for the loop body of a loop $\$$ expression that occurs either at the top level or in the body of a definition. In particular, the loop body should return a single, nonstobj value. As usual, such requirements are relaxed when the loop $\$$ expression occurs in a theorem. For example, the following form is legal, and in fact is admitted by ACL2, even though the loop\$ expression is illegal both at the top level and in any function body.

(thm (equal (loop\$ for $\mathrm{x}$ in ' (A B C) collect (mv $\mathrm{x} x$ ))

' $((A \quad A)(B \quad B)(C \quad C))))$ 


\section{Reasoning with Loop\$}

We recall the definition of sum-squares above and state a simple theorem about that function.

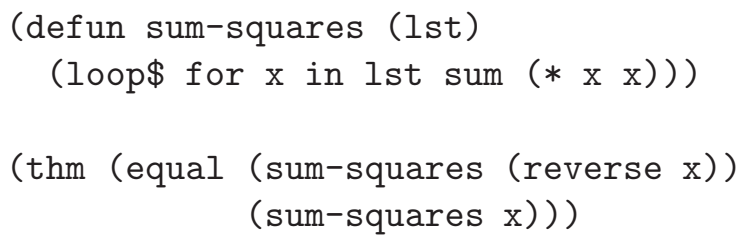

The proof fails, but the experienced ACL2 user quickly completes the proof by noticing terms of the form (sum\$ FN (revappend X Y)) in the checkpoints and then proving the following lemma.

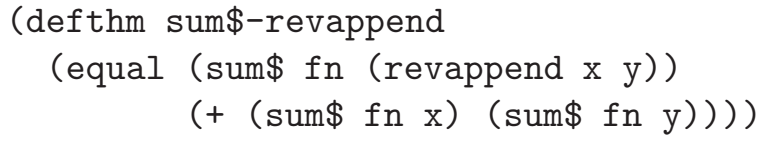

Of course, one could instead define sum-squares recursively and go through a similar process to find and prove a suitable lemma about (sum-squares (revappend $\mathrm{x} y$ )), and then prove the thm. But by using loop\$, one needn't prove such a lemma more than once. For example, suppose we define:

(defun sum-cubes (lst)

(loop\$ for $\mathrm{x}$ in lst sum $(* \mathrm{x} \mathrm{x} \mathrm{x}))$ )

Then the following theorem is proved automatically by applying the lemma above, sum $\$$-revappend.

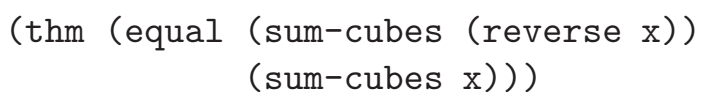

If instead sum-cubes were defined by recursion, then one would need first to prove a new lemma about (sum-cubes (revappend $\mathrm{x}$ y)).

We have seen that reasoning about loop $\$$ expressions generally reduces to reasoning about loop $\$$ scions. The community book projects/apply/loop, included in the book projects/apply/top that is typically included when reasoning about apply $\$$ or loop $\$$, has helpful lemmas to support automation of reasoning about loop\$. Perhaps over time, the lemma sum\$-revappend (above) and other useful lemmas will be included in lemma libraries for reasoning about loop\$. Also see community book projects/apply/report. lisp for examples of reasoning with (close analogues of) loop\$ scions.

Let's look at one more proof example.

(thm (equal (sum-squares ' (1 $\left.\begin{array}{llll}1 & 2 & 3 & 4\end{array}\right)$ ) 30)

This proves automatically using these rules.

Rules: ( (:EXECUTABLE-COUNTERPART EQUAL)

$$
\text { ( : EXECUTABLE-COUNTERPART SUM-SQUARES) ) }
$$

Thus, evaluation involving loop $\$$ expressions (in this case, in the body of the definition of sum-squares) can take place during proofs. This simple example does not require warrants, but those are handled with evaluation as well; see Section 6.3 ,

The community book projects/apply/mempos.lisp may be helpful for reasoning about loop $\$$ expressions that have more than one iteration variable or an UNTIL clause. 


\section{Evaluation}

We have seen that loop $\$$ calls are translated into logic by generating calls of loop $\$$ scions. Thus, evaluation of loop $\$$ calls can take place by evaluating those translations. Indeed, that is what happens when loop $\$$ expressions occur at the top level, as we discuss in the first subsection below. However, when loop $\$$ expressions are in guard-verified code, their evaluation generally reduces to efficient evaluation of corresponding Common Lisp loop expressions, as we discuss in the second subsection below. We next discuss a key exception: during proofs, loop\$ scions are always used for evaluation, with a twist: there is accounting for warrants. Finally we discuss performance.

\subsection{Evaluation in the top-level loop}

We first consider evaluation of loop\$ calls taking place directly in the top-level loop (rather than within a function body). Every top-level form is evaluated by first translating into logic. In particular, evaluation of a loop $\$$ call in the top-level loop takes place by translating into logic, that is, into calls of loop $\$$ scions as described in Section 4. The following example shows this behavior in action.

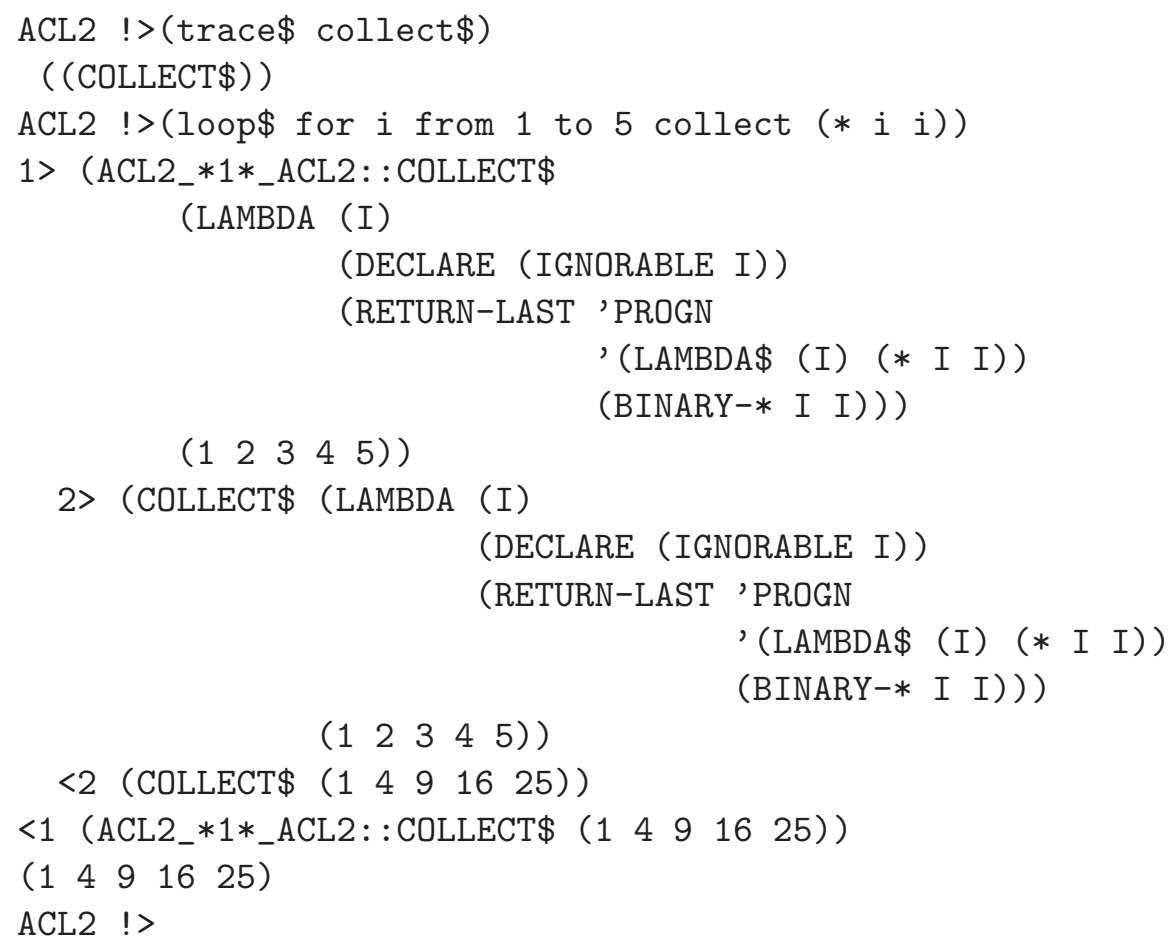

Top-level evaluation treats all warrants as being true at the top level. In short, the reason is that at the top level, attachments may be used (see :DOC defattach), and all warrants have true attachments. Thus for example, if we introduce the function symbol square as defined in Section 3 and we replace the body of the loop $\$$ expression above by (square $i$ ), then we get the same evaluation result.

\subsection{Guards and fast Common Lisp evaluation for function calls}

Recall that guard verification permits ACL2 to execute with Common Lisp definitions. When this is done, loop $\$$ expressions are computed by evaluating corresponding Common lisp loop expressions. For example, recall this definition from Section 3 . 


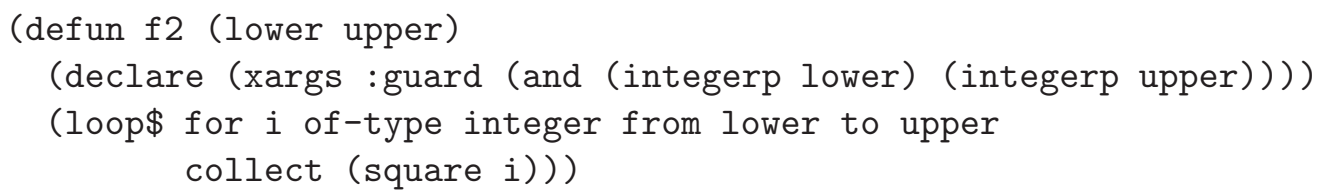

If we evaluate (f2 1 5), we get the same result as in the preceding example: (l $\left.\begin{array}{lllll}1 & 4 & 9 & 16 & 25\end{array}\right)$. However, if we first trace collect $\$$, as before, then this time we see no calls of collect $\$$. The reason is clear from the single-step Common Lisp macroexpansion of the loop\$ expression displayed below, which shows that when attachments are allowed (i.e., *aokp* is true), as is the case when evaluating directly in the top-level loop, then the corresponding loop expression is evaluated. (We'll discuss the other case when we discuss evaluation during proofs.)

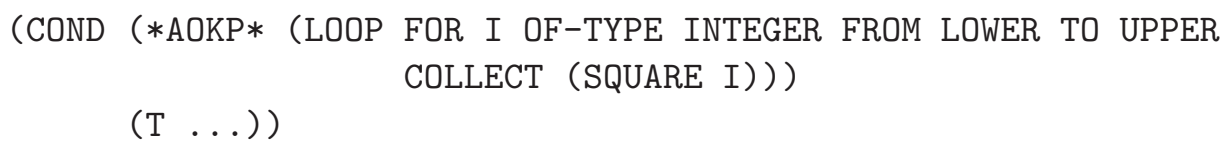

Guard verification is necessary before a loop\$ call will be evaluated using loop in Common Lisp. Indeed, if we instead define $\mathrm{f} 2$ by adding the xargs declaration :verify-guards nil, then evaluation of ( $f 215$ ) will show collect $\$$ calls in a trace as displayed in the preceding subsection. The natural question is then: What are the guard proof obligations generated for a loop $\$$ expression?

Let us begin by considering our $\mathrm{f} 2$ example. In that case, ACL2 reports the following "non-trivial part of the guard conjecture".

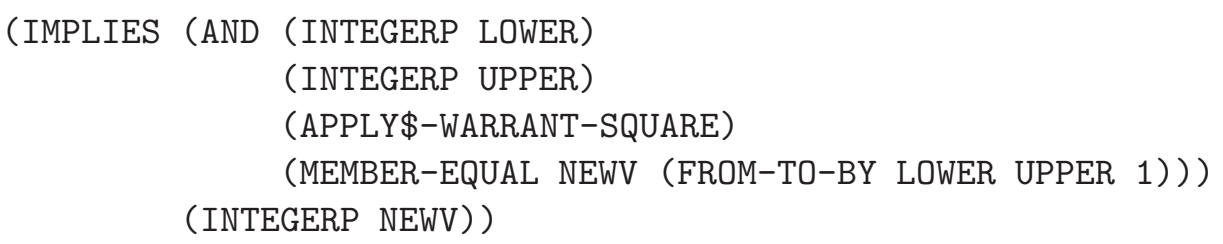

This formula states that if newv is a member of the indicated from-to-by expression, then newv is an integer. It is generated by the expression " $i$ of-type integer" in the loop $\$$ expression, which is necessary for guard verification of the loop body, since the guard of (square $n$ ) is (integerp $n$ ).

The formula displayed above illustrates the first of the following three classes of guard proof obligations arising from loop $\$$ expressions. The second one below is a rather obvious requirement. The third arises because of Common Lisp quirks: all tails may be checked in the ON case, including nil, and the type-checks for indices from $i$ to $j$ by $k$ may include one step past the point where iteration stops.

- Special Conjecture (a): Every member of the target list satisfies the guard of the function object.

- Special Conjecture (b): On every member of the target list, the function object produces a result of the right type: a number if the loop operator is SUM and a true list if the operator is APPEND.

- Special Conjectures (c): For (loop\$ ... ON ...), nil satisfies the type expression (if any). For a (FROM-TO-BY $i j k$ ) target list: $i, j, k$, and $(+i(* k(f l o o r(-j i) k)$ ) k) each satisfy the type expression (if any).

Warrant hypotheses can be relevant to guard verification, although the user is generally spared from thinking about that. Consider the following variant of $\mathrm{f} 2$ above, where SUM replaces COLLECT.

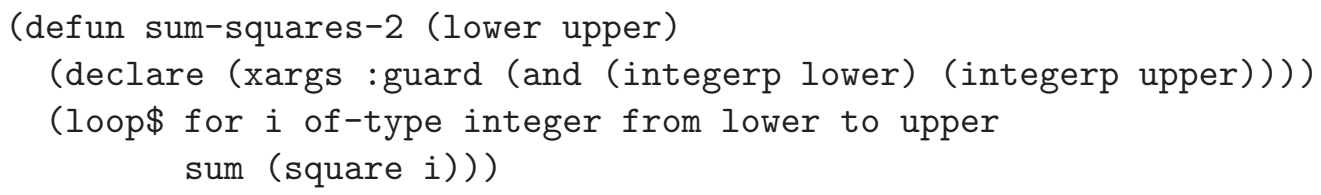


The hypothesis (warrant square) does not appear in the :guard, but it is added automatically in the generated guard conjecture. This addition is necessary; the proof fails without it. This addition is also justified: the Special Conjectures are generated only to avoid guard violations when evaluating the Common Lisp loop expression that corresponds to the loop\$ expression, and that only happens when attachments are permitted (see the use of *aokp* in the Common Lisp definition of loop $\$$ above). But when attachments are permitted then all warrant hypotheses are true, which justifies them as hypotheses.

We conclude this subsection by noting that instead of using the of-type construct, one can supply a : guard as indicated in Section 2. The loop $\$$ expression in the body of $f 2$ could instead be written as follows, giving an equivalent definition.

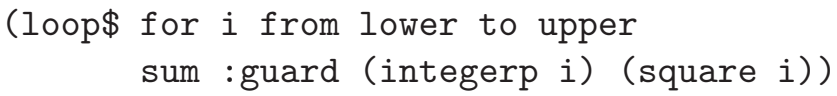

The of-type construct may provide for more efficient Common Lisp code, since the : guard construct is ignored by the compiler. On the other hand, the : guard construct is more flexible, since it can reference more than one variable.

\subsection{Evaluation during proofs}

An example in Section 5 shows that evaluation of loop $\$$ expressions can take place during proofs. That example did not require any warrant hypotheses, but in general they might be required.

As noted above, when attachments are allowed then all warrant hypotheses hold, so ACL2 evaluates (apply $\$, F \ldots$ ) by simply calling $F$ on the supplied arguments. Unfortunately, attachments are not allowed during proofs.

However, a major design goal for ACL2 is fast evaluation during proofs. That includes calls of apply $\$$, calls of loop $\$$, and calls of functions that lead to calls of apply $\$$ or loop $\$$. Thus, a challenge is for ACL2 to support evaluation of (apply $\${ }^{\prime} F \ldots$. by simply calling $F$, as discussed above for the case that attachments are allowed.

ACL2 meets this challenge, as clearly illustrated by its ability to prove the following theorem using evaluation of a loop\$ expression (where sum-squares-2 is defined as in the preceding subsection).

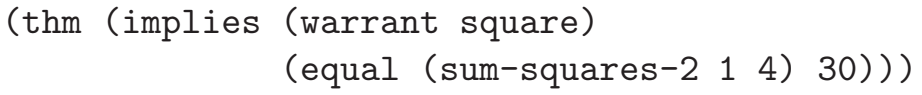

Let us inspect the summary.

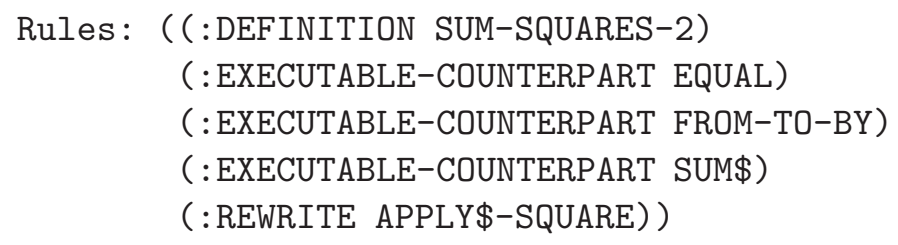

The use of the rule (:rewrite apply\$-square) is expected, as discussed in Section 5 , The use of the definition rule for sum-squares-2 (as opposed to evaluation of that function's call) is perhaps surprising: it takes place early, in the preprocess step of the waterfall, where evaluation of loop $\$$ expressions that require warrants is not supported. The simplifier then evaluates the body of sum-squares-2 with respect to the binding alist ( (upper. '4) (lower. '1)). The hints shown below avoid the definition rule but the proof still succeeds by running the executable counterpart of sum-squares-2, which actually checks that the necessary warrant is available as a hypothesis. 


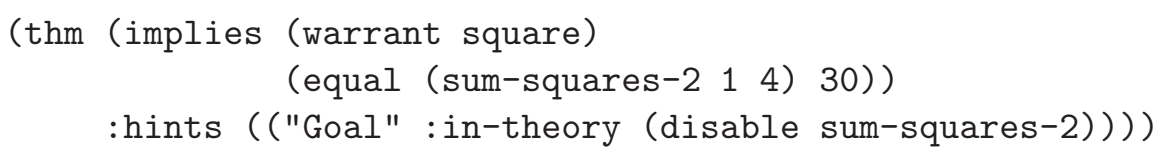

Deleting the hypothesis causes the proof to fail after the missing warrant is forced by proof-time evaluation.

For more examples involving evaluation of loop $\$$ expressions during proofs, see the community books system/tests/apply-in-proofs.lisp and system/tests/loop-tests.lisp.

We conclude our discussion of evaluation during proofs by remarking on the implementation and its impact on performance. In the preceding subsection we discussed the macroexpansion of loop $\$$ expressions in Common Lisp when attachments are allowed. We now consider the other case, which invokes the usual translation to a call of a loop\$ scion. The expansion is as follows for the loop\$ expression in the definition of $\mathrm{f} 2$.

(COND (*AOKP*; Attachments are allowed.

(LOOP FOR I OF-TYPE INTEGER FROM LOWER TO UPPER COLLECT (SQUARE I)))

(T (COLLECT\$ ' (LAMBDA (I)

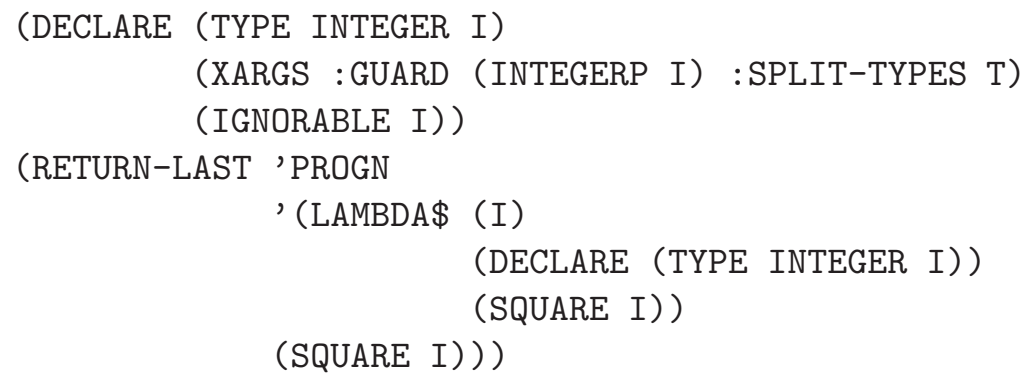

The loop $\$$ scion call is generated by finding a term associated with the loop\$ expression in the world.

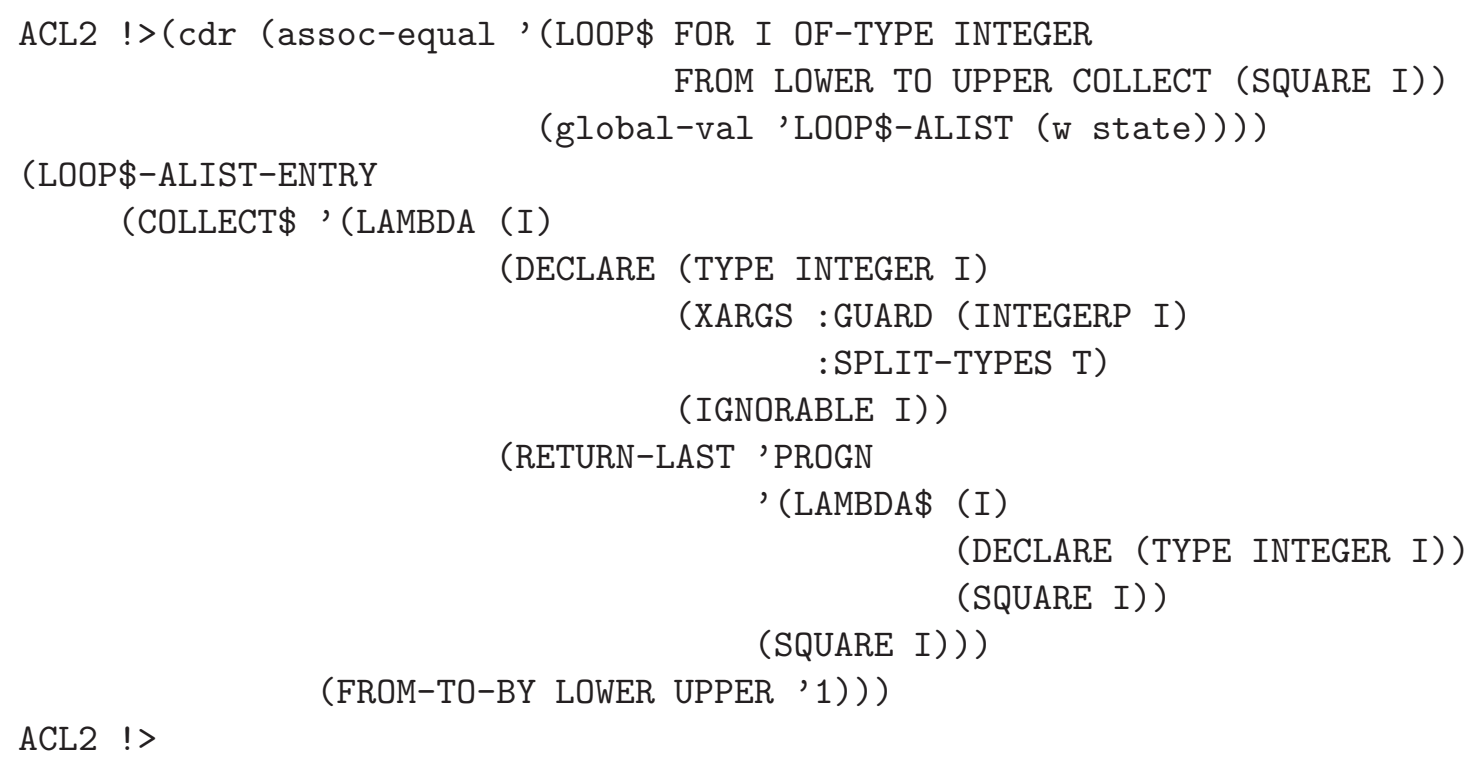

ACL2 updates the loop\$-alist structure at the conclusion of a defun event (here, for $f 2$ ), by collecting all tagged loops (RETURN-LAST 'PROGN '(LOOP\$ ...) $\quad e$ ) and associating each such (LOOP\$ ...) 
with its corresponding translation, $e$. Well, almost: the problem is that $e$ might not be executable, in particular because $\underline{\mathrm{mv}-\text { let }}$ calls are eliminated by translation using $\underline{\mathrm{mv}-\mathrm{nth}}$ calls. So the implementation (with function convert-tagged-loop\$s-to-pairs) converts the loop $\$$ translation to executable code (with function logic-code-to-runnable-code), notably by inserting calls of mv-list.

A more complete answer also takes into account early loading of compiled files and removes ACL2specific guard information. Here is the definition of loop\$ (from the ACL2 sources) in Common Lisp.

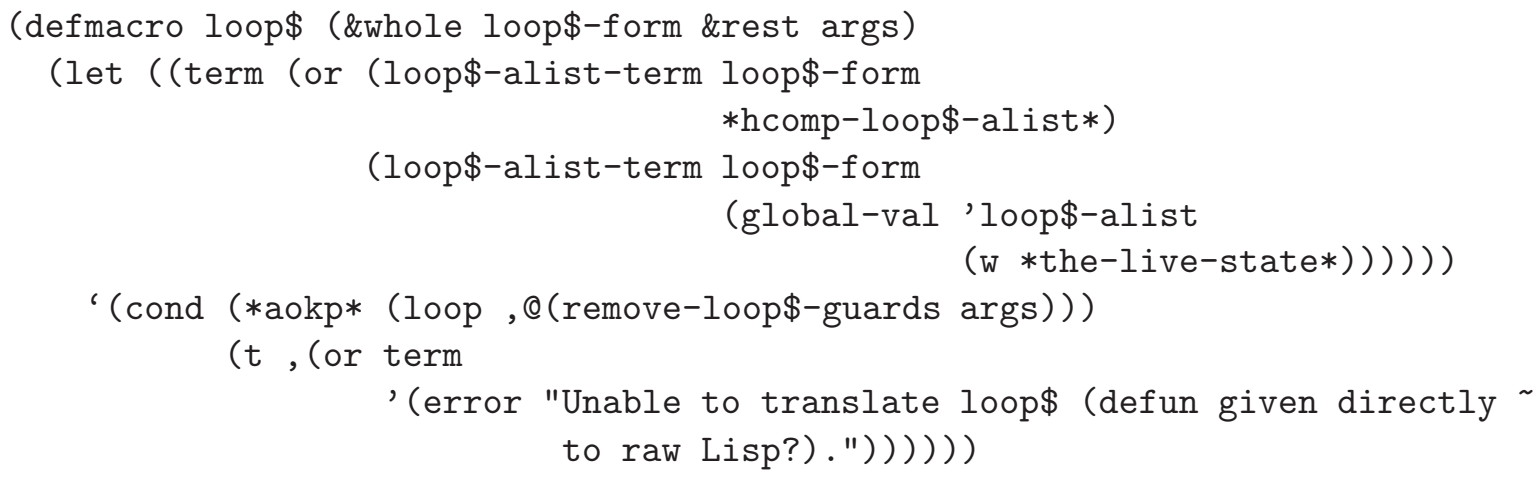

The following two issues are raised by this method of evaluating loop $\$$ expressions during proofs.

- Section 3 noted the necessity of warrant hypotheses because attachments are disallowed during proofs. Specifically, as we have seen, calls of loop\$ scions may ultimately lead to calls of apply $\$$ on user-defined functions, which is where warrant hypotheses are required. The implementation permits evaluation of such apply $\$$ calls during rewriting but forces the necessary warrant hypotheses that are not known in the current rewriting context (the so-called 'type-alist ').

- Evaluation of loop\$ expressions can be considerably slower during proofs than it is at the top level, because of the use of loop $\$$ scions and apply $\$$ rather than evaluation of a Common Lisp loop expression. We considered allowing this faster evaluation during proofs, but that would require checking (or forcing) the warrant hypothesis of every user-defined function in the loop body (and also the WHEN and UNTIL clauses, if present), even for functions that are rarely called when evaluating the loop body, such as in the error branch of an IF expression.

\subsection{Performance}

The results reported in the comments below were produced using an ACL2 version (git hash 5eb79e7697) built on CCL on April 4, 2018, running on a 3.5 GHz 4-core Intel(R) Xeon(R) with Hyper-Threading. Times in seconds are realtime; also shown are bytes allocated. The key take-away is the comparison between (c) and (d): Evaluation in the ACL2 loop, including the integer-listp call in the guard, is very close in time to the corresponding evaluation directly in Common Lisp.

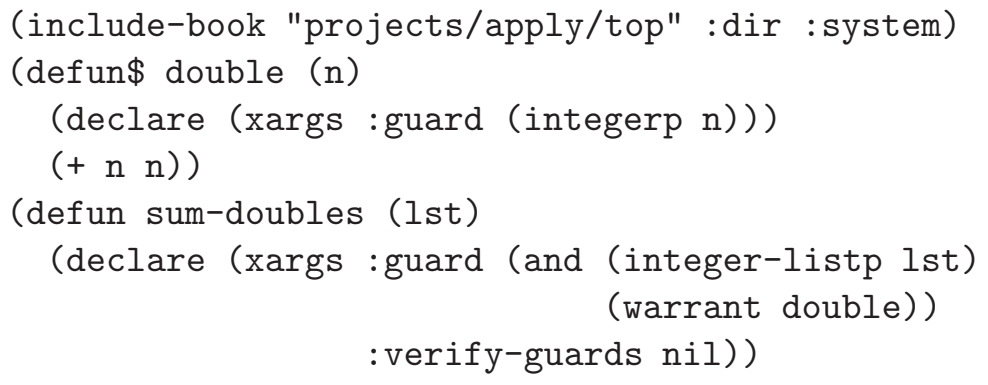




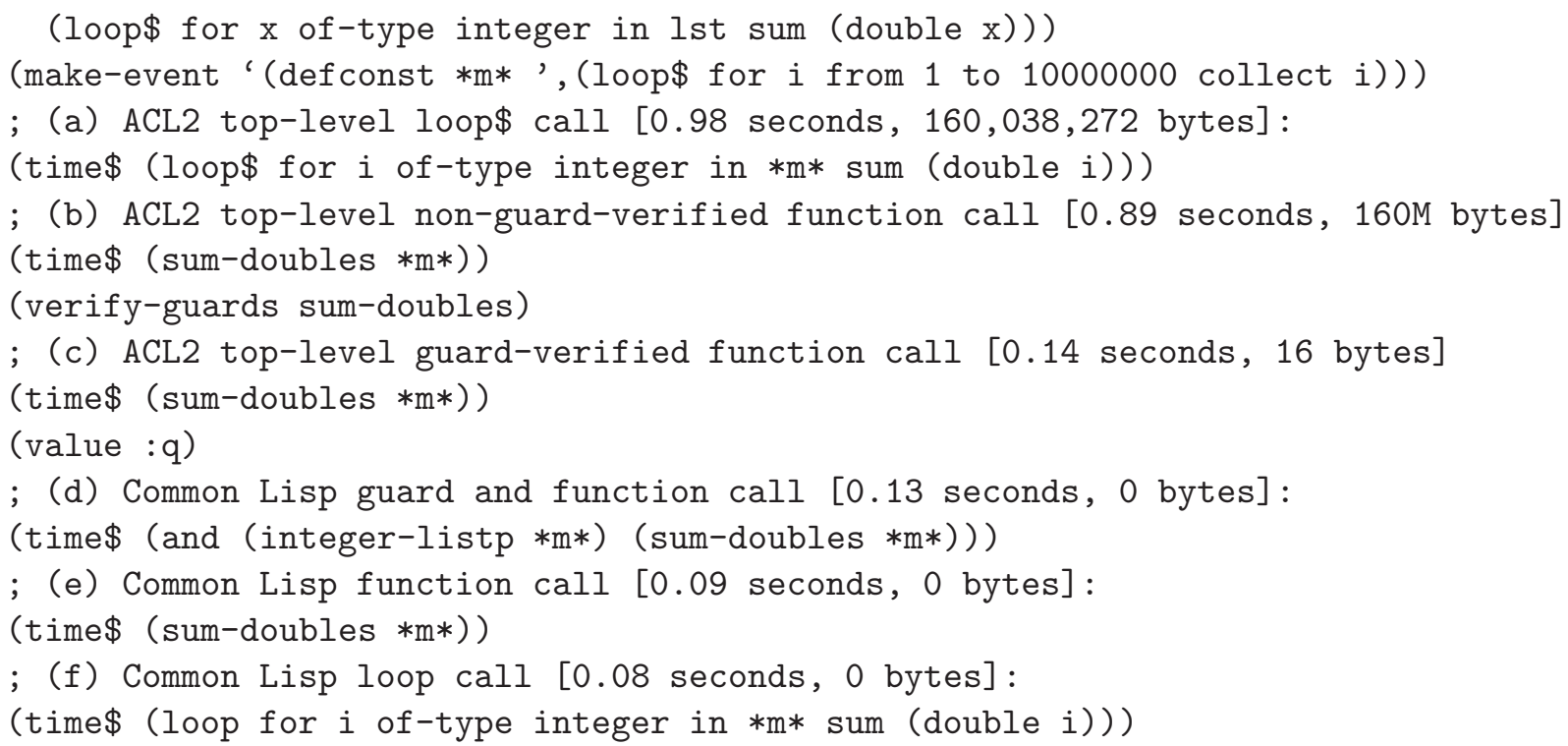

\section{Limitations, Future Work, and Conclusion}

We know of several limitations that may be addressed in future work. For more information search for "Possible Future Work on Loop\$" in ACL2 source file translate.lisp.

- Reliance on apply $\$$ and scions means that quoted lambda objects may appear in formulas that, when entered by the user, had no such objects in them. This can cause trouble because two obviously equivalent quoted lambda objects may be distinct objects. This arises especially when a theorem statement may involve a loop $\$$ iteration variable with a different name than used in some function definition. E.g., ' (lambda ( $\mathrm{x}$ ) ( $\mathrm{sq} \mathrm{x}$ )) is a different object than ' (lambda (y) (sq y)) even though they are obviously functionally equivalent. Declare forms in such objects can also render them unnecessarily distinct. While the functional equivalence of lambda objects is, of course, undecidable, trivial cases like these ought to be caught by the prover but are not. Furthermore, because they are so obvious the user may overlook the differences! Until these issues are smoothed out in the prover, checkpoints involving lambda objects must be read with unusual care!

- Apply $\$$ only works on logic mode functions so it is an error if a program mode function appears in a loop $\$$ body or a WHEN or UNTIL clause.

- Apply $\$$ does not work on state- or stobj-using functions, hence neither does loop $\$$. This may be very difficult to change.

- At the time this paper was submitted, recursion within a loop\$ body was not supported and so it is not described here. But since then we have added support for it; see :DOC loop\$-recursion.

- As noted at the end of Subsection 6.3, evaluation of a loop\$ expression uses loop\$ scions instead of the more efficient Common Lisp loop when either during a proof or directly in the top-level loop (rather than in a function body). (Of course, such evaluation can be expected to be much faster than term rewriting.) For the top-level case, perhaps ACL2 should report an error and insist on the use of the utility top-level, as illustrated by the following example.

ACL2 !>(time\$ (loop\$ for i from 1 to 10000000 sum i)) 


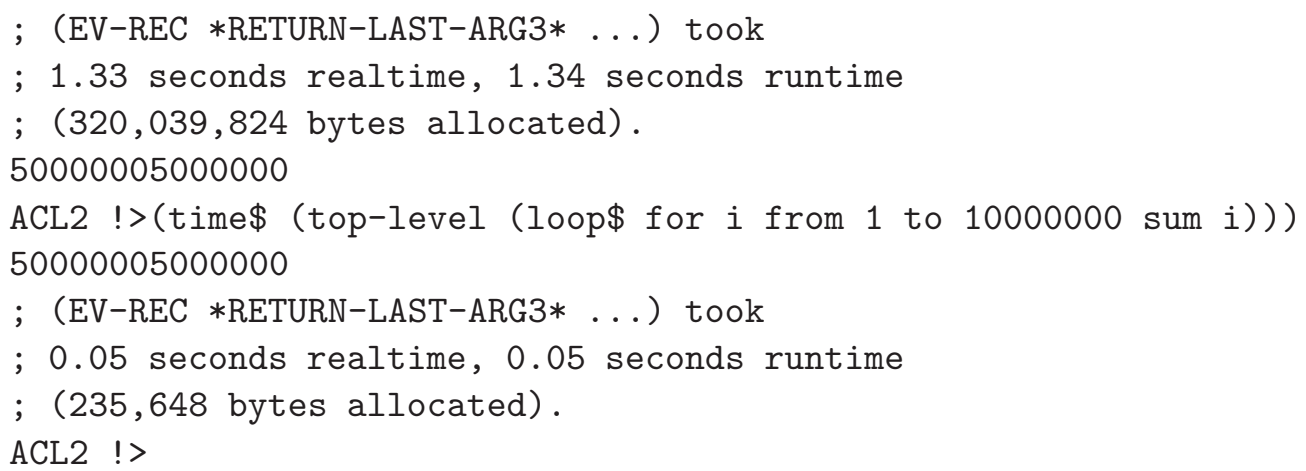

- Common Lisp loop supports more general forms than loop\$, some of which are feasible to support in loop\$. Here are a couple of examples.

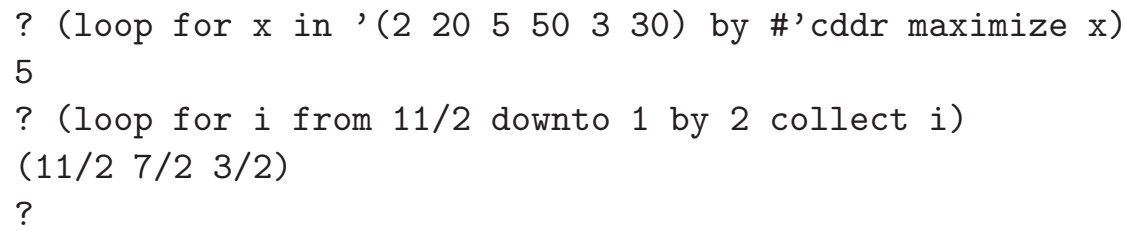

Despite these limitations, we have seen that loop\$ provides efficient execution and can make reasoning more succinct. We expect to evolve its implementation as users tell us what most needs improvement.

\section{Acknowledgments}

This material is based upon work supported in part by DARPA under Contract No. FA8650-17-1-7704. We are also grateful for support of this work by ForrestHunt, Inc., and for helpful reviewer feedback.

\section{References}

[1] R. Boyer \& J S. Moore (1988): The Addition of Bounded Quantification and Partial Functions to a Computational Logic and Its Theorem Prover. Journal of Automated Reasoning 4(2), pp. 117-172, doi:10.1007/BF00244392. See http://www.cs.utexas.edu/users/moore/publications/quant.pdf.

[2] R. S. Boyer \& J S. Moore (1997): A Computational Logic Handbook, Second Edition. Academic Press, New York.

[3] M. Kaufmann \& J S. Moore (2018): Limited Second-Order Functionality in a First-Order Setting. Journal of Automated Reasoning, doi:10.1007/s10817-018-09505-9. Available at http://www.cs.utexas.edu/users/kaufmann/papers/apply/.

[4] M. Kaufmann, J S. Moore \& The ACL2 Community (2020): The Combined ACL2+Books User's Manual. http://www.cs.utexas.edu/users/moore/acl2/manuals/current/manual/index.html.

[5] K. Kunen (1998): Nonconstructive Computational Mathematics. J. Autom. Reason. 21(1), pp. 69-97, doi $10.1023 / \mathrm{A}: 1005888712422$

[6] J S. Moore (1975): Introducing Iteration into the Pure LISP Theorem-Prover. IEEE Transactions on Software Engineering 1(3), pp. 328-338, doi:10.1109/TSE.1975.6312857, See http://www.cs.utexas.edu/users/moore/publications/parc-csl-tr-74-3.pdf 